\title{
BLOCH SPACE AND THE NORM OF THE BERGMAN PROJECTION
}

\author{
Antti Perälä \\ University of Helsinki, Department of Mathematics and Statistics \\ P.O. Box 68, FI-00014 Helsinki, Finland; antti.i.perala@helsinki.fi
}

\begin{abstract}
In this note we calculate the norm of the Bergman projection from the space of essentially bounded functions to the Bloch space. We complement author's earlier results in [6] and their generalizations in [5].
\end{abstract}

\section{Introduction}

Bergman projection is no doubt a central object in the study of analytic function spaces. In the setting of the unit disk $\mathbf{D}$, it arises as the following integral operator

$$
\operatorname{Pf}(z)=\int_{\mathbf{D}} \frac{f(w) d A(w)}{(1-z \bar{w})^{2}} .
$$

For $0<p<\infty$ we denote by $A^{p}$ the Bergman spaces, consisting of $L^{p}$-integrable analytic functions on $\mathbf{D}$ with respect to the normalized area measure $d A(z)=\pi^{-1} d x d y$ for $z=x+i y$. By $H^{\infty}$ we mean the space of bounded analytic functions on the disk.

When $p=2, P$ is an orthogonal projection, and it is obvious that the norm of $P: L^{2} \rightarrow A^{2}$ is one. However, this projection is also bounded $L^{p} \rightarrow A^{p}$ for $1<p<\infty$, but unbounded for $p=1$ and $p=\infty$.

There is a natural space, onto which $P$ maps $L^{\infty}$ boundedly. This is the Bloch space $\mathcal{B}$, which consists of analytic functions $f$ such that

$$
\|f\|_{*}=\sup _{z \in \mathbf{D}}\left(1-|z|^{2}\right)\left|f^{\prime}(z)\right|<\infty
$$

holds true. In [6] the author showed that

$$
\|P f\|_{*} \leq(8 / \pi)\|f\|_{\infty}
$$

where the constant $8 / \pi$ is the best possible.

Note that the quantity $\|\cdot\|_{*}$ defined above is only a semi-norm. It clearly fails to distinguish constant functions, so it cannot be a norm. The most common way to overcome this inconvenience is to set

$$
\|f\|=\|f\|_{*}+|f(0)|
$$

to be the norm on $\mathcal{B}$.

In this note, we complement the result of [6]. We prove that

$$
\|P f\| \leq(1+8 / \pi)\|f\|_{\infty},
$$

doi:10.5186/aasfm.2013.3850

2010 Mathematics Subject Classification: Primary 30H30, 32A25.

Key words: Bergman projection, Bloch space, operator norm.

The author acknowledges support by the Academy of Finland project no. 75166001. 
where the constant in the inequality is the best possible. The reader is referred to [8] for more information about analytic Bergman and Bloch spaces. It is fairly easy to see that the presented method works also for the more general setting in [5]; this will be commented in the last section.

We remark that calculating the exact norm of $P$ on $L^{p}$ spaces is a long-standing problem and only partial results are known, see [1] and [7]. Similar questions have been solved in the setting of Hardy and Segal-Bargmann spaces in the papers [3] and [2], respectively. Also, the paper [4] deals with a related question for the Beurling transform.

\section{The result}

Denote by $\|P\|$ the operator norm of $P: L^{\infty} \rightarrow \mathcal{B}$, that is, the optimal constant $C$ in the inequality

$$
\|P f\| \leq C\|f\|_{\infty}
$$

We will show that $\|P\|=1+8 / \pi$. We will begin with simple lemma, which was actually proven in the recent paper by Kalaj and Markovic [5].

Lemma 2.1. The norm of the projection $P$ satisfies $\|P\| \leq 1+8 / \pi$.

Proof. Just note that $|P f(0)|=\left|\int_{\mathbf{D}} f(z) d A(z)\right| \leq\|f\|_{\infty}$.

In view of this lemma, it is enough to prove $\|P\| \geq 1+8 / \pi$. The problem is then, whether the quantities $\|P f\|_{*}$ and $|P f(0)|$ can be maximized simultaneously. We will answer this problem in the affirmative.

The method in [6] uses functions

$$
g_{z}(w)=\frac{w|1-z \bar{w}|^{3}}{|w|(1-\bar{z} w)^{3}},
$$

which can be applied to maximize $\|P f\|_{*}$. Here, these do not seem to work, because $\left|P g_{z}(0)\right|<1$ for all $z$. The next proposition is proven in [6], but we sketch the proof for the reader's convenience.

Proposition 2.2. The optimal constant $C$ for

$$
\|P f\|_{*} \leq C\|f\|_{\infty}
$$

is $8 / \pi$. Moreover, it is obtained as the limit of

$$
\left(1-|z|^{2}\right)\left|\left(P g_{z}\right)^{\prime}(z)\right|=\int_{\mathbf{D}} \frac{2\left(1-|z|^{2}\right)|w| d A(w)}{|1-z \bar{w}|^{3}}=F(z)
$$

as $|z| \rightarrow 1^{-}$.

Proof. Given $z \in \mathbf{D}$, the quantity $\left(1-|z|^{2}\right)\left|(P g)^{\prime}(z)\right|$ over $\|g\|_{\infty} \leq 1$ is maximized by $g_{z}$. This is transparent from the integral formula

$$
\left|(P g)^{\prime}(z)\right|=\left|\int_{\mathbf{D}} \frac{2 \bar{w} g(w) d A(w)}{(1-z \bar{w})^{3}}\right|
$$

which is obtained by differentiating inside the integral.

Denote by $\varphi_{z}(w)=(z-w) /(1-\bar{z} w)$ the usual Möbius transformation of the disk. By applying a change of variables $w \mapsto \varphi_{z}(w)$, one gets

$$
\int_{\mathbf{D}} \frac{2\left(1-|z|^{2}\right)|w| d A(w)}{|1-z \bar{w}|^{3}}=\int_{\mathbf{D}} \frac{2|z-w| d A(w)}{|1-z \bar{w}|^{2}} .
$$


From this, one sees that $F(z)$ is subharmonic. Since it is easy to verify that $F(z)$ is also radial, the maximum of $F(z)$ is obtained as $|z| \rightarrow 1^{-}$. But

$$
F(1)=\int_{\mathbf{D}} \frac{2 d A(w)}{|1-w|}=8 / \pi
$$

and the proof is complete.

The key to the proof of our result is noting that only the behaviour of $g_{z}$ near the boundary matters. Define new test functions $g_{z}^{r}$ with $\left\|g_{z}^{r}\right\|_{\infty} \leq 1$ as follows:

$$
g_{z}^{r}(w)= \begin{cases}g_{z}(w) & \text { if }|w| \geq r \\ 1 & \text { if }|w| \leq r^{2}\end{cases}
$$

Finally, define $g_{z}^{r}$ on $\left\{r^{2}<|w|<r\right\}$ so that $g_{z}^{r}$ is continuous on $\overline{\mathbf{D}}$.

Lemma 2.3. For every $r \in(0,1)$, we have

$$
\left(1-|z|^{2}\right)\left(P g_{z}^{r}\right)^{\prime}(z) \rightarrow 8 / \pi
$$

as $|z| \rightarrow 1^{-}$.

Proof. Because $\left|g_{z}(w)-g_{z}^{r}(w)\right| \leq 2$ on $\mathbf{D}$ and $\left|g_{z}(w)-g_{z}^{r}(w)\right|=0$ when $|w|>r$, we can estimate

$$
\left(1-|z|^{2}\right)\left|\left(P\left(g_{z}-g_{z}^{r}\right)\right)^{\prime}(z)\right| \leq \int_{B(0, r)} \frac{4\left(1-|z|^{2}\right)|w| d A(w)}{|1-z \bar{w}|^{3}} .
$$

The right hand side goes to 0 as $|z| \rightarrow 1^{-}$. Therefore, one can conclude that

$$
\left(1-|z|^{2}\right)\left(P g_{z}^{r}\right)^{\prime}(z) \rightarrow 8 / \pi
$$

as $|z| \rightarrow 1^{-}$.

We have now collected sufficient amount of information to prove our main result without much trouble.

Theorem 2.4. The operator norm of $P: L^{\infty} \rightarrow \mathcal{B}$ is $1+8 / \pi$.

Proof. For every $z \in \mathbf{D}$, we have

$$
\left|P g_{z}^{r}(0)\right| \geq r^{4}-\int_{\mathbf{D} \backslash B\left(0, r^{2}\right)} d A(w)=2 r^{4}-1 \rightarrow 1
$$

as $r \rightarrow 1$. Given $\epsilon>0$, we may pick $r>0$ such that $\left|P g_{z}^{r}(0)\right|>1-\epsilon / 2$ for every $z \in \mathbf{D}$. Fix such $r$. According to the previous lemma, one can pick $z \in \mathbf{D}$ such that

$$
\left(1-|z|^{2}\right)\left(P g_{z}^{r}\right)^{\prime}(z)>8 / \pi-\epsilon / 2 .
$$

But, one then ends up with a function $g_{z}^{r}$ such that

$$
\|P\| \geq\left\|P g_{z}^{r}\right\| \geq\left|P g_{z}^{r}(0)\right|+\left(1-|z|^{2}\right)\left|\left(P g_{z}^{r}\right)^{\prime}(z)\right|>1+8 / \pi-\epsilon .
$$

Therefore $\|P\| \geq 1+8 / \pi$, which together with Lemma 2.1 proves the claim.

Denote by $\mathcal{B}_{0}$ the little Bloch space consisting of $f \in \mathcal{B}$ such that

$$
\lim _{|z| \rightarrow 1^{-}}\left(1-|z|^{2}\right)\left|f^{\prime}(z)\right|=0 .
$$

We equip $\mathcal{B}_{0}$ also with the norm $\|\cdot\|$ used for the Bloch space. It is known that $P: C(\overline{\mathbf{D}}) \rightarrow \mathcal{B}_{0}$ is bounded and onto, where $C(\overline{\mathbf{D}})$ stands for the functions continuous 
on $\overline{\mathbf{D}}$. Note that $g_{z}^{r} \in C(\overline{\mathbf{D}})$, by definition. This shows that the operator norm of $P: C(\overline{\mathbf{D}}) \rightarrow \mathcal{B}_{0}$ is likewise $1+8 / \pi$.

\section{Several dimensions}

In their recent paper [5], Kalaj and Markovic prove a generalization of [6]. This paper can currently be found in arXiv. Denote by $\mathbf{B}_{n}$ the unit ball of $\mathbf{C}^{n}$, and let $\alpha>-1$. On $\mathbf{B}_{n}$ we use the following measure

$$
d V_{\alpha}=c_{\alpha}\left(1-|z|^{2}\right)^{\alpha} d V(z) .
$$

Here $d V$ is the normalized $2 n$-dimensional Lebesgue measure on $\mathbf{B}_{n}$, and $c_{\alpha}$ is a constant such that $d V_{\alpha}$ is a probability measure.

The Bloch space $\mathcal{B}_{n}$ on $\mathbf{B}_{n}$ the set of those holomorphic functions $f$ on such that

$$
\|f\|_{*, n}=\sup _{z \in \mathbf{B}_{n}}\left(1-|z|^{2}\right)|\nabla f(z)|<\infty,
$$

where $\nabla=\left(\partial_{z_{1}}, \partial_{z_{2}}, \ldots, \partial_{z_{n}}\right)$ is the usual complex gradient. The little Bloch space $\mathcal{B}_{0, n}$ is defined analogously to the case $n=1$. The Bergman projection $P_{n, \alpha}$ is defined as

$$
P_{n, \alpha} f(z)=\int_{\mathbf{B}_{n}} \frac{f(w) d V_{\alpha}(w)}{(1-\langle z, w\rangle)^{1+n+\alpha}} .
$$

Here

$$
\langle z, w\rangle=\sum_{i=1}^{n} z_{i} \bar{w}_{i}
$$

is the inner product of $\mathbf{C}^{n}$. Denote by $\Gamma$ the Euler Gamma function. According to [5], the optimal constant $C(\alpha, n)$ in

$$
\left\|P_{n, \alpha}\right\|_{*, n} \leq C(\alpha, n)\|f\|_{\infty}
$$

satisfies

$$
C(\alpha, n)=\frac{\Gamma(2+n+\alpha)}{\Gamma^{2}((2+n+\alpha) / 2)},
$$

which equals $8 / \pi$ if $\alpha=0$ and $n=1$, so it generalizes the main result of [6].

Of course $\mathcal{B}_{n}$ can be equipped with the norm

$$
\|f\|_{n}=\|f\|_{*, n}+|f(0)|,
$$

so we can discuss the operator norm of $P_{n, \alpha}$, which we denote by $\|P\|_{n, \alpha}$.

Like in [6], the authors of [5] use functions

$$
g_{z}(w)=\frac{w_{1}}{\left|w_{1}\right|} \frac{|1-\langle z, w\rangle|^{n+2+\alpha}}{(1-\langle w, z\rangle)^{n+2+\alpha}}
$$

to obtain $C(\alpha, n)$ as the limit of

$$
\left(1-|z|^{2}\right)\left|\nabla\left(P g_{z}\right)(z)\right|
$$

when $z \rightarrow e_{1}=(1,0, \ldots, 0)$. By defining the functions $g_{z}^{r}$ analogously to the previous section, one obtains

Corollary 3.1. The operator norm of $P_{n, \alpha}$ from $L^{\infty}\left(\mathbf{B}_{n}\right)$ onto $\mathcal{B}_{n}$ equals $C(\alpha, n)+$ 1. The same is true about $P_{n, \alpha}: C\left(\overline{\mathbf{B}_{n}}\right) \rightarrow \mathcal{B}_{0, n}$.

The details of the proof are left as exercise for the reader. The proof is completely analogous to the case $n=1$ and $\alpha=0$. 


\section{References}

[1] Dostanic, M.: Two sided norm estimate of the Bergman projection on $L^{p}$ spaces. - Czechoslovak Math. J. 58:2(133), 2008, 569-575.

[2] Gryc, W., and T. Kemp: Duality in Segal-Bargmann spaces. - J. Funct. Anal. 261:6, 2011, 1591-1623.

[3] Hollenbeck, B., and I. Verbitsky: Best constants for the Riesz projection. - J. Funct. Anal. $175: 2,2000,370-392$.

[4] IwAnIEC, T.: The best constant in a BMO-inequality for the Beurling-Ahlfors transform. Michigan Math. J. 33:3, 1986, 387-394.

[5] Kalaj, D., and M. Markovic: Norm of the Bergman projection. - Math. Scand. (to appear), arXiv:1203.6009.

[6] PerÄLä, A.: On the optimal constant for the Bergman projection onto the Bloch space. - Ann. Acad. Sci. Fenn. Math. 37, 2012, 245-249.

[7] ZHu, K.: A sharp norm estimate of the Bergman projection on $L^{p}$ spaces. - Contemp. Math. 404, 2006, 199-205.

[8] ZHu, K.: Operator theory in function spaces. 2nd edition. - Math. Surveys Monogr. 138, Amer. Math. Soc., Providence, RI, 2007.

Received 28 February 2013 • Accepted 3 May 2013 